\title{
Prevalência de estomias de eliminação em uma microrregião do norte de Minas Gerais
}

\author{
Prevalence of elimination stomas in a microregion in the north of Minas Gerais \\ Prevalencia de ostomías de eliminación en una micro-región del norte de Minas Gerais
}

Elisangela Ribeiro Barros ${ }^{1}$, Eline Lima Borges ${ }^{1}$, Célia Maria de Oliveira $^{1}$

ORCID IDS

Barros ER (iD https://orcid.org/0000-0001-7590-7019

Borges EL iD https://orcid.org/0000-0002-0623-5308

Oliveira CM (D) https://orcid.org/0000-0002-1937-7364
COMO CITAR

Barros ER; Borges EL; Oliveira CM. Prevalência de estomias de eliminação em uma microrregião do norte de Minas Gerais. ESTIMA, Braz. J. Enterostomal Ther., 16:e3418. https://doi.org/10.30886/ estima.v16.654 PT

\begin{abstract}
RESUMO
Objetivos: Estimar a prevalência de pessoas com estomia de eliminação e caracterizá-las quanto aos aspectos sociodemográficos e clínicos. Método: Estudo transversal descritivo com amostra de 27 pacientes com estomias de eliminação, residentes em uma microrregião do norte de Minas Gerais, que atenderam aos critérios de inclusão. Os dados coletados foram registrados em instrumento utilizado em estudos anteriores e analisados por meio de estatística descritiva. Estudo aprovado pelo Comitê de Ética. Resultados: A prevalência de pessoas com estomia foi de 2,16/10.000, a amostra continha 55,6\% de homens, 40,7\% eram casados, com média de idade de 59,1 anos, 59,2,\% eram alfabetizados e 74,0\% tinham baixa renda; 59,3\% das estomias decorreram de câncer, sendo mais frequentes os de cólon e reto, 70,4\% das estomias eram definitivas e 74,0\% eram colostomias. Dermatite foi a principal complicação e $68,2 \%$ dos pacientes apresentavam efluente de consistência pastosa e realizavam autocuidado. Conclusão: A prevalência de pessoas com estomia foi semelhante à de outros municípios de Minas Gerais e abaixo do esperado pelas Associações Nacional e Internacional de Ostomizados. A caracterização das pessoas, das estomias e do manejo destas contribuirá para que profissionais de saúde e gestores revisem os protocolos de assistência utilizados na microrregião estudada.
\end{abstract}

DESCRITORES: Estomia; Estomas cirúrgicos; Cuidados de enfermagem; Perfil de saúde; Estomaterapia.

1 Universidade Federal de Minas Gerais - Escola de Enfermagem - Departamento de Enfermagem Ba Autora correspondente: Eline Lima Borges | Av. Professor Alfredo Balena, 190 - Santa Efigênia | CEP: 30.130-100 - Belo Horizonte/ MG - Brazil | E-mail: eborges@ufmg.br

Recebido: Out 122018 | Aceito: Dez 182018 


\begin{abstract}
Objectives: To estimate the prevalence of people with elimination stoma and to characterize them for sociodemographic and clinical aspects. Method: A descriptive cross-sectional study with a sample of 27 patients with elimination stomas residing in a microregion in the north of Minas Gerais, who met the inclusion criteria. The data collected were recorded in an instrument used in previous studies and analyzed using descriptive statistics. Study approved by the Ethics Committee. Results: The prevalence of people with stoma was 2.16/10.000, the sample contained $55.6 \%$ of men, $40.7 \%$ were married, mean age 59.1 years, 59.2\% were literate and $74.0 \%$ had low income; $59.3 \%$ of the stomas were cancerous, more frequent colon and rectum tumours, $70.4 \%$ of the stomas were definitive, and $74.0 \%$ were colostomies. Dermatitis was the main complication and $68.2 \%$ of the patients presented effluent of pasty consistency and performed self-care. Conclusion: The prevalence of people with stoma was like that of other municipalities in Minas Gerais and below that expected by the National and International Ostomized Associations. The characterization of the people, of the stomas and the management of these, will contribute for health professionals and managers to review the assistance protocols used in the studied microregion.
\end{abstract}

DESCRIPTORS: Stoma; Surgical stomas; Nursing care; Health profile; Stomatherapy.

\title{
RESUMEN
}

Objetivos: Estimar la prevalencia de personas con ostomía de eliminación y caracterizarlas con respecto a los aspectos sociodemográficos y clínicos. Método: Estudio transversal descriptivo con muestra de 27 pacientes con ostomías de eliminación, residentes en una micro-región del norte de Minas Gerais, que cumplieron los criterios de inclusión. Los datos recolectados fueron registrados en instrumento utilizado en estudios anteriores y analizados por medio de estadística descriptiva. Estudio aprobado por el Comité de Ética. Resultados: La prevalencia de personas con ostomía fue de 2,16/10.000, la muestra contenía 55,6\% de hombres, 40,7\% eran casados, con promedio de edad de 59,1 años, 59,2,\% eran alfabetizados y 74,0\% tenían baja renta; 59,3\% de las ostomías se derivaron de cáncer, siendo más frecuentes los de colon y recto, 70,4\% de las ostomías eran definitivas y 74,0\% eran colostomías. La dermatitis fue la principal complicación y 68,2\% de los pacientes presentaban efluente de consistencia pastosa y realizaban auto-cuidado. Conclusión: La prevalencia de personas con ostomía fue semejante a la de otros municipios de Minas Gerais y por debajo de lo esperado por las Asociaciones Nacional e Internacional de Ostomizados. La caracterización de las personas, de las ostomías y del manejo de estas contribuirá para que los profesionales de la salud y los gestores revisen los protocolos de asistencia utilizados en la micro-región estudiada.

DESCRIPTORES: Ostomía; Estomas quirúrgicos; Cuidados de enfermería; Perfil de salud; Estomaterapia..

\section{INTRODUÇÃO}

O vocábulo estoma ou estomia deriva do grego stoma e significa abertura. A estomia resulta de uma intervenção cirúrgica para estabelecer a comunicação entre uma víscera e o meio externo, de forma temporária ou permanente, com o objetivo de compensar o funcionamento prejudicado por alguma doença ou trauma. Uma estomia que tem a função de eliminação é denominada conforme a parte exteriorizada: ileostomia, colostomia e urostomia ${ }^{1}$.

A presença da estomia desencadeia mudanças significativas na vida dos indivíduos, dentre elas, a alteração da imagem corporal. A adaptação a essa nova imagem ocorre de acordo com as características emocionais, culturais e experiências anteriores de cada indivíduo ${ }^{2}$. Neste sentido, a inclusão social e a reabilitação das pessoas com estomia tornam-se tarefas primordiais para os profissionais de saúde e, especificamente, para o enfermeiro.
No Brasil, a assistência aos pacientes com estomia é realizado pelo Serviço de Atenção à Saúde das Pessoas Ostomizadas (SASPO). Esse serviço é classificado de acordo com a complexidade da assistência prestada e visa o atendimento integral, especializado e interdisciplinar à pessoa com estomia. No SASPO, os pacientes com estomia são cadastrados na Rede de Cuidados à Pessoa com Deficiência do Sistema Único de Saúde (SUS) e adquirem, mensalmente, os dispositivos e adjuvantes necessários ao cuidado da estomia. Esse serviço, em Minas Gerais, por meio da Secretaria de Estado, além de fornecer os equipamentos coletores e adjuvantes, deve realizar ações, por meio de uma equipe multidisciplinar, de orientação para o autocuidado e a prevenção de complicações relacionadas às estomias ${ }^{3}$.

$\mathrm{Na}$ região norte de Minas Gerais (Brasil), local do estudo, as desigualdades relacionadas às necessidades 
em saúde são semelhantes àquelas encontradas na região nordeste e no Vale do Jequitinhonha. Essas regiões apresentam necessidades de saúde acima da média geral observada no estado, compreendendo $14,0 \%$ da população do estado ${ }^{4}$.

Com o intuito de suprir parte dessas demandas relacionadas à saúde, a equipe de saúde dessa microrregião tem buscado melhorar a assistência aos pacientes com estomia, no SASPO I, potencializando esforços no sentido de aumentar os recursos humanos em número suficiente para prestar assistência multidisciplinar especializada a essas pessoas e adequar a estrutura física aos critérios preconizados pela Portaria SAS/MS no 400, de 16/11/2009. Por meio dessa iniciativa, objetiva-se reduzir as fragilidades das ações de saúde voltadas para reabilitação, orientação para o autocuidado, tratamento e prevenção das complicações relacionadas à estomia.

Neste contexto, a inexistência de estudos sobre a prevalência e a caracterização das pessoas com estomia dessa microrregião é um fator que prejudica o planejamento e as ações direcionadas a essa clientela. Vale ressaltar que, apesar dos esforços para atender às necessidades dos usuários desta microrregião, se faz necessário construir um novo modelo de assistência centrado no autocuidado.

\section{OBJETIVOS}

Considerando que a falta de informação do paciente sobre a estomia pode comprometer o autocuidado e que a não caracterização da pessoa com estomia constitui um obstáculo para o planejamento das ações e da gestão dos serviços de saúde, o estudo teve por objetivos estimar a prevalência de pessoas com estomia de eliminação e caracterizá-las quanto aos aspectos sociodemográficos e clínicos nessa microrregião de Minas Gerais.

\section{MÉTODO}

Trata-se de pesquisa descritiva, transversal, envolvendo pessoas com estomia de eliminação e visando a prevalência deste agravo em uma microrregião do norte de Minas Gerais.

O estudo foi realizado no SASPO I de Pirapora e envolveu também os seguintes municípios da microrregião do norte de Minas Gerais: Buritizeiro, Várzea da Palma, Ponto Chique, Ibiaí, Lassance e Santa Fé de Minas. A microrregião compreende cerca de $17.580 .000 \mathrm{~km}^{2}$ de extensão territorial e uma população estimada de 138.356 habitantes. Os municípios possuem distâncias variadas de Pirapora, sendo Buritizeiro o mais próximo, localizado a $8,1 \mathrm{~km}$, e Ponto Chique o mais afastado ${ }^{5}$, a $120,9 \mathrm{~km}^{2}$.

O SASPO I fica localizado no município-sede e é um serviço de referência, cobrindo toda a microrregião do norte de Minas Gerais. Apenas os municípios de Santa Fé de Minas e Lassance ainda não possuem pacientes com estomia cadastrados nesse serviço.

O estudo durou de janeiro de 2008 a novembro de 2017, correspondendo ao período de atendimento a essa clientela, por meio de um serviço de referência que dá cobertura à microrregião do norte de Minas Gerais.

Para participar do estudo, o paciente deveria possuir estomia de eliminação intestinal e/ou urinária, ter cadastro na Secretaria de Estado da Saúde, estar inserido no SASPO I e assinar o termo de Consentimento Livre e Esclarecido.

A identificação do participante se deu por meio da ficha existente no serviço e ele foi contactado por via telefônica. Neste processo, constatou-se que existiam 45 pacientes com estomia de eliminação, sendo que não havia pacientes dos municípios de Santa Fé de Minas e Lassance. Deste total, nove haviam falecido, oito tinham sido submetidos à cirurgia de reconstrução do intestino e quatro haviam mudado para outra região. Além destes, três pacientes estavam fora da cidade por tempo indeterminado e seis realizaram o cadastro após a confecção da estomia, durante o período de desenvolvimento do estudo. Sendo assim, 27 pessoas aceitaram participar da pesquisa, constituindo a amostra deste estudo.

A coleta de dados ocorreu no mês de dezembro de 2017 e foi realizada por um dos pesquisadores. Essa consistiu de entrevista e avaliação física do participante, incluindo a estomia, a pele ao redor e o dispositivo coletor em uso. Foram coletados dados referentes às variáveis socioeconômicas e demográficas, comportamentais e clínicas e esses foram registrados em um formulário que já foi utilizado em estudos anteriores realizados por este grupo de pesquisadores. Após essa etapa, os dados foram inseridos em um banco de dados organizado no programa Microsoft Office Excel®, versão 2013, e foram analisados por meio do método de estatística descritiva.

No cálculo de prevalência de pessoas com estomias de eliminação intestinais e urinárias na microrregião em estudo, no período de 2008 a 2017, considerou-se o número de usuários cadastrados no SASPO. Calculou-se o número de 
pacientes com estomia nesse serviço e este foi dividido pelo número de habitantes das cidades da microrregião pesquisada.

O estudo foi realizado com a aprovação do Comitê de Ética em Pesquisa, número 2.277.649, e a anuência do Secretário Municipal de Saúde de Pirapora, Minas Gerais. Os preceitos éticos estabelecidos na Resolução $\mathrm{n}^{\circ}$ 466, de 12 de dezembro de 2012, foram respeitados. Os participantes assinaram o termo de Consentimento Livre e Esclarecido, com a garantia de estarem cientes da pesquisa, do tempo a ser disponibilizado para realização de entrevista e avaliação clínica, bem como da divulgação e utilização dos dados para fins científicos.

\section{RESULTADOS}

A amostra do estudo foi constituída por 27 pessoas com estomia de eliminação, residentes em uma microrregião do norte de Minas Gerais. A prevalência encontrada foi de 2,16/10.000 pessoas nessa região. As variáveis socioeconômicas e demográficas (Tabela 1) e clínicas (Tabela 2) da amostra são apresentadas a seguir.

A maioria dos participantes do estudo informou residir em casa com saneamento básico (81,5\%). Em relação ao uso de cigarro, $85 \%$ dos participantes negaram tabagismo e $15 \%$ declararam que fumavam de 3 a 4 cigarros por dia.

A colostomia mostrou-se a mais prevalente dentre os tipos de estomias (74,0\%), as quais, em sua maioria, estavam localizadas na região do quadrante inferior esquerdo $(45,0 \%)$, em caráter definitivo $(65,0 \%)$ e eram de boca terminal $(45,0 \%)$. Houve predomínio de estomias de perfil baixo, com protrusão de até $1,5 \mathrm{~cm}(55,5 \%)$.

Os pacientes apresentaram efluente de consistência líquida $(27,3 \%)$, semipastosa $(4,6 \%)$ ou pastosa $(68,2 \%)$, com padrão de eliminação desde inúmeras vezes ao dia (41\%) até uma vez ao dia $(4,0 \%)$, passando por duas $(15 \%)$, três $(26,0 \%)$ e quatro a cinco $(15 \%)$ vezes ao dia. Eles relataram eliminação de flatos $(82,0 \%)$ com odor desagradável (52,0\%).

Dentre as doenças que desencadearam a confecção da estomia, a neoplasia apresentou predominância $(59,3 \%)$, sendo a neoplasia de reto a de maior destaque $(43,75 \%)$. As demais neoplasias localizavam-se no sigmoide $(18,75 \%)$, útero $(6,25 \%)$, colo do útero $(6,25 \%)$, ovário $(6,25 \%)$, bexiga $(12,5 \%)$ e próstata $(6,25 \%)$.

A maioria $(66,7 \%)$ dos pacientes apresentou complicações relacionadas à estomia devido à utilização incorreta do dispositivo e a outras variáveis clínicas, como índice de massa corporal. A dermatite foi a principal complicação encontrada na estomia $(47,6 \%)$ e na pele periestomal $(90,9 \%)$, sendo relacionada ao uso incorreto do dispositivo, como corte excessivo do orifício da barreira protetora com exposição da pele ao redor da estomia à ação do efluente. A hérnia paraestomal foi a segunda complicação mais encontrada (18,5\%).

Os dados demonstraram que a maior parcela das pessoas com estomia $(85,2 \%)$ tinha capacidade física para a realização dos cuidados com a estomia e o dispositivo. Entretanto, apenas $48,0 \%$ dos pacientes realizavam a troca do dispositivo e 52,0\% faziam a higienização da estomia.

Tabela 1. Aspectos socioeconômicos e demográficos $(n=27)$ dos pacientes com estomia de uma microrregião do norte de Minas Gerais, Brasil.

\begin{tabular}{|c|c|c|c|}
\hline Variáveis & Categorias & n (\%) & Média \\
\hline \multirow{2}{*}{ Sexo } & Feminino & $12(44,4)$ & - \\
\hline & Masculino & $15(55,6)$ & - \\
\hline \multirow{3}{*}{ Idade (anos) } & $<1$ & $1(3,7)$ & \multirow{3}{*}{59,1} \\
\hline & $43-59$ & $17(63,0)$ & \\
\hline & $67-82$ & $9(33,3)$ & \\
\hline \multirow{5}{*}{$\begin{array}{l}\text { Escolaridade } \\
\text { (anos de estudo) }\end{array}$} & 0 & $8(29,6)$ & \multirow{5}{*}{4,4} \\
\hline & 1 a 4 & $9(33,3)$ & \\
\hline & 5 a 8 & $3(11,1)$ & \\
\hline & 9 a 12 & $6(22,2)$ & \\
\hline & 13 a 17 & $1(3,7)$ & \\
\hline \multirow{2}{*}{ Alfabetização } & Analfabeto & $11(40,8)$ & - \\
\hline & Alfabetizado & $16(59,2)$ & - \\
\hline \multirow{6}{*}{ Estado civil } & Casado & $11(40,7)$ & - \\
\hline & União estável & $1(3,7)$ & - \\
\hline & Solteiro & $9(33,3)$ & - \\
\hline & Divorciado & $3(11,1)$ & - \\
\hline & Viúvo & $2(7,4)$ & - \\
\hline & Separado & $1(3,7)$ & - \\
\hline \multirow{3}{*}{ Cor/raça } & Branca & $7(25,9)$ & - \\
\hline & Preta & $6(22,2)$ & - \\
\hline & Parda & $14(51,9)$ & - \\
\hline \multirow{3}{*}{$\begin{array}{l}\text { Renda familiar mensal } \\
\text { (salário mínimo [SM] } \\
\text { R\$937,00) }\end{array}$} & $<1 \mathrm{SM}$ & $1(3,7)$ & \multirow{3}{*}{1,7} \\
\hline & 1 a $2 \mathrm{SM}$ & $19(70,3)$ & \\
\hline & 2 a $3 \mathrm{SM}$ & $7(25,9)$ & \\
\hline \multirow{2}{*}{ Aposentado } & Sim & $11(40,7)$ & - \\
\hline & Não* & $16(59,3)$ & - \\
\hline Total & & $27(100,0)$ & - \\
\hline
\end{tabular}


Tabela 2. Distribuição de frequência das variáveis clínicas ( $n=27$ ) dos pacientes com estomia de uma microrregião do norte de Minas Gerais, Brasil.

\begin{tabular}{|c|c|c|}
\hline Variáveis & Categorias & n (\%) \\
\hline \multirow{8}{*}{$\begin{array}{l}\text { Doença/situação } \\
\text { que levou à } \\
\text { cirurgia } \\
\text { de estomia }\end{array}$} & Neoplasia & $16(59,3)$ \\
\hline & Megacólon & $2(7,4)$ \\
\hline & $\begin{array}{l}\text { Ausência, atresia e estenose } \\
\text { congestiva do ânus }\end{array}$ & $1(3,7)$ \\
\hline & Fístula enterovesical & $1(3,7)$ \\
\hline & Bexiga neurogênica & $2(7,4)$ \\
\hline & Obstrução intestinal & $2(7,4)$ \\
\hline & Volvo sigmoide & $2(7,4)$ \\
\hline & Diverticulite & $1(3,7)$ \\
\hline \multirow{9}{*}{$\begin{array}{l}\text { Doenças } \\
\text { associadas }\end{array}$} & HAS & $9(33,3)$ \\
\hline & Cardiopatia & $1(3,7)$ \\
\hline & $\mathrm{DM}$ & $1(3,7)$ \\
\hline & DRC & $1(3,7)$ \\
\hline & Anemia & $1(3,7)$ \\
\hline & Doença de Chagas* & $1(3,7)$ \\
\hline & Doença mental* & $1(3,7)$ \\
\hline & Epilepsia & $1(3,7)$ \\
\hline & Não possuem & $12(44,4)$ \\
\hline \multirow{3}{*}{ Estado geral } & Bom & $26(96,3)$ \\
\hline & Regular & $1(3,7)$ \\
\hline & Ruim & $0(0)$ \\
\hline \multirow{4}{*}{ Locomoção } & Deambula & $25(92,6)$ \\
\hline & $\begin{array}{l}\text { Deambula com ajuda de } \\
\text { prótese/órtese }\end{array}$ & $0(0)$ \\
\hline & Não deambula & $1(3,7)$ \\
\hline & Cadeirante & $0(0)$ \\
\hline \multirow{5}{*}{ IMC } & Baixo peso $(<18,5)$ & $2(7,4)$ \\
\hline & Normal $(18,5-24,9)$ & $9(33,3)$ \\
\hline & Sobrepeso $(25-29,9)$ & $11(40,8)$ \\
\hline & Obesidade $(\geq 30)$ & $4(14,8)$ \\
\hline & $\begin{array}{l}\text { Não se aplica } \\
\text { (não há IMC para criança < 2) }\end{array}$ & $1(3,7)$ \\
\hline
\end{tabular}

\section{DISCUSSÃO}

A temática sobre estomia de eliminação vem ganhando relevância devido ao crescente número de cirurgias que desencadeiam sua confecção. No Brasil, isso se deve ao aumento de doenças crônicas não transmissíveis relacionadas à maior expectativa de vida das pessoas, bem como ao aumento da violência urbana e dos acidentes de trânsito ${ }^{6}$.
A quantidade de estudos sobre estomias vem crescendo e estes buscam contribuir para a melhoria do desempenho dos profissionais envolvidos no cuidado e no planejamento das ações em saúde. Eles visam, sobretudo, direcionar políticas de saúde que contemplem essa clientela.

A prevalência de pessoas com estomia identificada na microrregião do estudo encontra-se abaixo da estabelecida pela Associação Internacional de Ostomizados (IAO) e pela Associação Brasileira de Ostomizados (ABRASO). Entretanto, esse resultado é semelhante ao encontrado em estudo desenvolvido em outros municípios de Minas Gerais. Tal situação pode estar relacionada à falta de conhecimento dos usuários da microrregião em estudo sobre o SASPO, uma vez que a avaliação diagnóstica dos SASPO em Minas Gerais revelou que poucas pessoas recebem atendimento pelos programas estaduais e municipais ${ }^{7}$.

A avaliação sociodemográfica e econômica demonstra o predomínio de pessoas com estomia do sexo masculino, resultado também descrito em estudos desenvolvidos em outras regiões brasileiras ${ }^{8}$. Este dado pode estar relacionado à maior exposição de indivíduos do sexo masculino a traumas, doenças e agravos, bem como à menor frequência destes nos serviços de prevenção e promoção à saúde. Tal postura é descrita como fator que dificulta o diagnóstico e o tratamento precoce dos agravos e doenças que levam à confecção da estomia".

Os pacientes com estomia dessa microrregião são, na sua maioria, aposentados, pensionistas, beneficiários ou desenvolvem trabalhos com baixa remuneração, constituindo uma clientela com baixa renda. Esses resultados estão presentes em estudo de diferentes regiões do $\mathrm{Brasil}^{10}$. Deve-se considerar que na região do norte de Minas Gerais, juntamente com o Vale do Jequitinhonha, há um grande número de municípios com os maiores níveis de pobreza do estado ${ }^{11}$.

Estudos anteriores comprovam que o nível de escolaridade possui associação com o autocuidado ${ }^{6,9}$. Vale ressaltar que o nível de escolaridade exerce influência sobre a capacidade de compreensão do indivíduo no que se refere à sua saúde. $\mathrm{O}$ elevado índice de analfabetismo impacta na qualidade de vida dos pacientes, na sua capacidade de buscar assistência e garantir seus direitos 5 .

Neste sentido, o analfabetismo de uma parcela significativa dos pacientes constitui um fator preocupante, 
uma vez que o menor grau de instrução dificulta o acesso ao conhecimento sobre a doença, podendo tornar o paciente com estomia passivo e dependente, sentindo-se incapaz de executar o autocuidado e, sobretudo, prejudicando seu processo de reabilitação ${ }^{12,13}$.

Compreende-se que essa capacidade de aquisição do conhecimento em saúde motiva o indivíduo a buscar de forma eficiente recursos para o autocuidado, a manutenção, a promoção e a recuperação da saúde ${ }^{14}$. Assim, é responsabilidade do profissional desenvolver habilidades de alfabetização em saúde, buscando ultrapassar as barreiras e enfatizar as potencialidades apresentadas pelo paciente, oferecendo a este condições para alcançar a sua autonomia.

Reconhecer a importância da educação é passo essencial para o aprimoramento da prática de enfermagem e torna-se uma importante ferramenta na manutenção, prevenção e promoção da saúde do indivíduo com estomia. A problematização e a produção conjunta do conhecimento concretizam-se como um exercício de autonomia e potencializam o cuidado e o autocuidado ${ }^{15}$.

A maioria dos participantes relatou desconforto e constrangimento devido aos gases, com odor desagradável, provenientes das eliminações intestinais involuntárias. Esses desajustes de ordem fisiológica propiciam alterações psicológicas e espirituais nas pessoas com estomia, impactando de forma negativa na sua qualidade de vida ${ }^{2}$.

Em relação às causas de indicação das estomias, a neoplasia obteve maior destaque, com predomínio de tumores de cólon e reto. No Brasil, o câncer de cólon e reto é uma das principais causas cirúrgicas para a confecção de estomia ${ }^{7,10,16}$. Esse tipo de câncer está relacionado à obesidade, situação observada neste estudo ${ }^{17}$.

As complicações clínicas mais relevantes observadas no estudo estavam relacionadas ao uso incorreto do dispositivo, como exemplo, o corte excessivo do orifício da barreira protetora, sendo a dermatite a complicação de maior destaque. Estudos semelhantes demonstram que a dermatite é a principal complicação cutânea apresentada pelos pacientes com estomia ${ }^{8,18}$

O ganho de peso foi um fator preocupante observado no estudo, uma vez que ele está relacionado a complicações como retração, prolapso e hérnia paraestomal. Considerando que parte significativa dos participantes da pesquisa apresentou sobrepeso e obesidade e que a hérnia paraestomal foi a segunda complicação mais apresentada pelas pessoas com estomia, o acompanhamento e a assistência profissional são de extrema importância para prevenção dessas complicações ${ }^{18,19}$.

Em relação à altura da estomia, merece destaque o grande número de pacientes que possuem estomia baixa e na altura da pele. Esta situação pode levar à dificuldade de adesão do dispositivo, podendo aumentar o risco de descolamento e ocorrência de dermatite, evidenciada neste estudo. Neste sentido, é necessário o uso de maior quantidade de produtos e/ou de dispositivo convexo e/ ou cinto, elevando o custo do cuidado a esses usuários ${ }^{8}$.

Observou-se uma quantidade significativa de estomias localizadas no quadrante superior do abdome, localização não habitual e inadequada. A localização adequada da estomia é um fator determinante para higienização e adaptação do dispositivo coletor, proporciona melhor adesão desse dispositivo, evitando o extravasamento de efluentes com a pele periestomal, e previne possíveis complicações. Nesse sentindo, a demarcação da estomia é de extrema importância para a promoção do autocuidado, facilitando a reinserção social da pessoa com estomia ${ }^{18,20}$.

A realização da demarcação pelo enfermeiro estomaterapeuta, no Brasil, vem ganhando destaque e o envolvimento dos enfermeiros nas produções científicas vem aumentando, em relação aos demais países da América Latina. Esses estudos, realizados em diversas regiões do país, evidenciam a importância do enfermeiro na prática desse procedimento, contribuindo de forma relevante para o aumento da qualidade de vida dos pacientes com estomia. A atuação especializada desse profissional a partir do pré-operatório, além de direito do paciente, reduz a ocorrência de complicações de estomia e pele periestomal e diminui a ansiedade e o medo do paciente, possibilitando uma melhoria significativa no processo de reabilitação. Essa conduta que ratifica a importância do conhecimento diferenciado do estomaterapeuta nessa prática e na gestão dos cuidados às pessoas com estomia também é compartilhada por países desenvolvidos, como a Irlanda ${ }^{20-23}$.

Alguns pacientes relataram que o procedimento de manejo do dispositivo foi orientado por profissional de enfermagem. Esse fato estimula a discussão sobre o conhecimento dos profissionais de enfermagem em relação ao cuidado com o paciente com estomia e a importância da educação permanente desses profissionais para garantir uma assistência segura e eficaz ${ }^{24}$. Estudo realizado em um 
hospital público da capital do país sugere a implementação do processo de enfermagem como forma de sistematizar o cuidado. Por meio da consulta de enfermagem, o enfermeiro avalia sua própria assistência e melhora a qualidade do cuidado aos pacientes com estomia ${ }^{25}$. Outras produções científicas também evidenciaram a sistematização da assistência de enfermagem como um instrumento facilitador na reabilitação da pessoa com estomia, de forma a minimizar o sofrimento e estimular o autocuidado, garantindo a integralidade da assistência prestada a esses pacientes ${ }^{1}$.

Deve-se ressaltar que os pacientes não eram avaliados periodicamente, o que pode ter ocasionado as complicações clínicas de estomia e periestomia verificadas no estudo. Evidenciou-se em estudo recente que as complicações podem ser prevenidas por meio de avaliação frequente dos pacientes, no intuito de identificar fatores de risco que possam gerar complicações ${ }^{18}$. Isso demonstra a importância do acompanhamento do paciente pelo enfermeiro ${ }^{26}$. Esse profissional é capaz de proporcionar o cuidado e estimular o autocuidado. Tais medidas são imprescindíveis para a reabilitação do paciente com estomia ${ }^{12,13}$.

Vale destacar que o estudo teve como limitação a dificuldade de deslocamento dos pacientes com estomia à sede do SASPO nessa microrregião. Essa dificuldade, evidenciada principalmente nos pacientes de zona rural, constitui uma barreira para a utilização dos serviços de saúde e pode comprometer a qualidade da assistência prestada. Isso remete a uma reflexão em relação à distribuição da referência entre os municípios ${ }^{8}$.

\section{CONCLUSÃO}

A prevalência de pessoas com estomia identificada foi coerente com a encontrada em alguns municípios de Minas Gerais e está abaixo do estabelecido pela IAO e pela ABRASO. A maioria dos participantes tinha poucos anos de estudo e uma parcela significativa era analfabeta. Houve predominância de pessoas casadas, do sexo masculino, com nível econômico baixo, índice alterado de massa corporal, em bom estado geral e com independência para deambular.

A colostomia foi o tipo mais frequente de estomia. A neoplasia foi o principal motivo para confecção de estomia, com maior destaque para a neoplasia de reto. A dermatite foi a principal complicação da estomia e da região periestomal e estava relacionada ao uso incorreto do dispositivo. Outros estudos sobre esta temática deverão ser desenvolvidos, buscando estimular reflexões sobre a importância da integralidade do cuidado, do autocuidado e de ações preventivas e de detecção precoce de patologias e agravos que levam à confecção de estomias.

\section{CONTRIBUIÇÃO DOS AUTORES}

Conceitualização, Barros ER e Oliveira CM; Metodologia, Barros ER; Oliveira CM e Borges EL; Investigação, Barros ER e Oliveira CM; Redação - Primeira versão, Barros ER e Oliveira CM; Redação - Revisão \& Edição, Borges EL; Supervisão, Borges EL.

\section{REFERÊNCIAS}

1. Jesus PBR, Sena MN, Bispo NO, Alves PS, Santos DM. Sistematização da assistência de enfermagem às pessoas com estomias intestinais: revisão integrativa. ESTIMA, Braz J Enterostomal Ther. 2018;16:e1718. https://doi.org/10.30886/ estima.v16.418 PT

2. Coelho AR, Santos FS, Poggetto MTD. A estomia mudando a vida: enfrentar para viver. REME Rev Min Enferm. 2013;17(2):258-67. $\quad$ https://doi.org/10.5935/14152762.20130021

3. Brasil. Ministério da Saúde. Secretaria de Atenção à Saúde. Dispõe sobre as Diretrizes Nacionais para a Atenção à Saúde das Pessoas Ostomizadas no âmbito do Sistema Único de Saúde. Portaria n. 400, 16 novembro 2009. Brasília, 2009.

4. Ferreira Junior S, Fahel MCX, Horta CJG, Diniz JS. Desigualdades nas necessidades em saúde entre os municípios do estado de
Minas Gerais: uma abordagem empírica no auxílio às políticas públicas. Rev Administração Pública e Gestão Social (APGS). 2017;9(2):75-134.

5. Instituto Brasileiro de Geografia e Estatística. Censo Demográfico 2010. Características da população e dos domicílios: resultados do universo [Internet]. 2010 [citado out. 2017]. Disponível em: https://ww2.ibge.gov.br/home/ estatistica/populacao/censo2010/caracteristicas da populacao/tabelas pdf/tab1.pdf

6. Lenza NFB, Sonobe HM, Buetto LS, Santos MG, Lima MS. O ensino do autocuidado aos pacientes estomizados e seus familiares: uma revisão integrativa. Rev Bras Promoç Saúde. 2013;26(1):139-45. https://doi.org/10.5020/18061230.2013. p139 
7. Moraes JT, Assunção RS, Sá FS, Lessa ER, Corrêa LS. Perfil de pessoas estomizadas de uma região de saúde mineira. Enferm Foco. 2016;7(2):22-26. https://doi.org/10.21675/2357707X.2016.v7.n2.788

8. Freitas JPC; Borges EL; Bodevan EC. Caracterização da clientela e avaliação de serviço de atenção à saúde da pessoa com estomia de eliminação. ESTIMA, Braz J Enterostomal Ther. 2018;16:e0918. https://doi.org/10.30886/estima.v16.402 PT

9. Miranda SM, Luz MHBA, Sonobe HM, Andrade EMLR, Moura ECC. Caracterização sociodemográfica e clínica de pessoas com estomia em Teresina. ESTIMA, Braz J Enterostomal Ther. 2016;14(1):29-35. https://doi.org/10.5327/z18063144201600010005

10. Aguiar JC, Pereira APS, Galisteu KJ, Lourenção LG, Pinto MH. Aspectos sociodemográficos e clínicos de estomizados intestinais provisórios. REME Rev Min Enferm. 2017;21:e1013. https://doi.org/10.5935/1415-2762.20170023

11. Oliveira DBO, Fernandes EA. A inter-relação entre pobreza e meio ambiente para os municípios de Minas Gerais. Rev Economia e Agronegócio (REA). 2017;15(1):1614-79.

12. Almeida EJ, Silva AL. Caracterização do Perfil Epidemiológico dos Estomizados em Hospitais da Secretaria de Estado de Saúde do Distrito Federal. ESTIMA, Braz J Enterostomal Ther. 2015;13(1):6-11. $\quad$ https://doi.org/https://doi.org/10.5327/ z1806-3144201500010004

13. Luz ALA, Luz MHBA, Antunes A, Oliveira GS, Andrade EMLR, Miranda SM. Perfil de los pacientes ostomizados: revisión integrativa de la literatura. Cultura de los Cuidados. 2014;18(39):115-23. https://doi.org/10.7184/cuid.2014.39.13

14. Fung CSC, Yu EYT, Guo VY, Wong CKH, Kung K, Ho SY, et al. Development of a Health Empowerment Programme to improve the health of working poor families: protocol for a prospective cohort study in Hong Kong. BMJ Open. 2016;6(2):e010015. https://doi.org/10.1136/ bmjopen-2015-010015

15. Gazzinelli MF, Souza V, Fonseca RMGS, Fernandes MM, Carneiro ACLLC, Godinho LK. Práticas educativas grupais na atenção básica: padrões de interação entre profissionais, usuáriose conhecimento. Rev Esc Enferm USP. 2017;49(2):28491. https://doi.org/10.1590/50080-623420150000200014

16. Valle TD, Turrini RNT, Poveda VB. Fatores intervenientes para o início do tratamento de pacientes com câncer de estômago e colorretal. Rev Latino-Am Enferm. 2017;25:e2879. https:// doi.org/10.1590/1518-8345.1493.2879
17. Sena JF, Medeiros LP, Melo MDM, Souza AJG, Freitas LS, Costa IKF. Perfil de estomizados com diagnóstico de neoplasias cadastrados em uma associação. Rev Enferm UFPE on line. 2017;11(Suppl 2):873-80.

18. Pinto IES, Queirós SMM, Queirós CDR, Silva CRR, Santos CSVB, Brito MAC. Fatores de risco associados ao desenvolvimento de complicações do estoma de eliminação e da pele periestomal. Rev Enf Ref. 2017;15(4):155-66. https://doi. org/10.12707/RIV17071

19. Wound, Ostomy and Continence Nurses Society (WOCN). Pediatric ostomy complications: best practice for clinicians. Mount Laurel: NJ, WOCN Society; 2016.

20. Silva JC, Borsatto AZ, Teixeira ER, Umpiérrez AF. Demarcação abdominal por enfermeira estomoterapêuta. Enfermería: Cuidados Humanizados. 2017;6(1):12. https://doi. org/10.22235/ech.v6i1.1365

21. 21. Teles AAS, Eltink CF, Martins LM, Lenza NFB, Sasaki VDM, Sonobe HM. Mudanças físicas, psicossociais e os sentimentos gerados pela estomia intestinal para o paciente: revisão integrativa. Rev Enferm UFPE on line. 2017;11(Suppl 2):106272.

22. Bonill-delas Nieves C, Capilla DíazC,Celdrán-Mañas M, MoralesAsencio JM, Hernández-Zambrano SM, Hueso-Montoro C. Percepção de pacientes ostomizados sobre os cuidados de saúde recebidos. Rev Latino-Am Enferm. 2017;25:e2961. https://doi.org/10.1590/1518-8345.2059.2961

23. Freitas $\mathrm{RCL}$, Resende JL, Rodrigues $\mathrm{PL}$, Magalhães $\mathrm{MO}$, Carmo IC, Resende MA, et al. Importância da atuação do enfermeiro na demarcação do estoma no pré-operatório mediato. Rev Eletrônica Acervo Saúde. 2018;(Suppl 11):S1567-S1573. https://doi.org/10.25248/REAS173 2018

24. Falkenberg MB, Mendes TPL, Moraes EP, Souza EM. Educação em saúde e educação na saúde: conceitos e implicações para a saúde coletiva. Ciência \& Saúde Coletiva. 2014;19(3):847-52. https://doi.org/10.1590/1413-81232014193.01572013

25. Monteiro SNC, Kamada I, Silva AL, Souza TCR. Perfil de crianças e adolescentes estomizados atendidos de um hospital público do Distrito Federal. ESTIMA, Braz J Enterostomal Ther. 2017;12(3):23-32.

26. Vasconcellos FM, Xavier ZDM. O enfermeiro na assistência do cliente colostomizado baseado na teoria de Orem. Rev Recien. 2015;5(14):25. https://doi.org/10.24276/ rrecien2358-3088.2015.5.14.25-37 